\title{
ADVANCES IN NONLINEAR ANALYSIS
}

\section{EDITORS-IN-CHIEF}

Vicentiu Radulescu, Craiova

Marco Squassina, Brescia

\section{HONORARY EDITORS}

Viorel Barbu, Iasi

Haim Brezis, Piscataway, NJ

Philippe G. Ciarlet, Hong Kong

Jean Mawhin, Louvain-la-Neuve

Roger Temam, Bloomington, IN

Michel Willem, Louvain-la-Neuve

\section{EDITORIAL BOARD}

Shair Ahmad, San Antonio, TX

Thomas Bartsch, Giessen

Vieri Benci, Pisa

Franco Brezzi, Pavia

Marco Degiovanni, Brescia

Manuel del Pino, Santiago

Guido De Philippis, Trieste

Frank Duzaar, Erlangen
Donato Fortunato, Bari

Nicola Fusco, Napoli

Marius Ghergu, Dublin

Jacques Giacomoni, Pau

Olivier Goubet, Amiens

Louis Jeanjean, Besançon

Jan Kristensen, Oxford

Irena Lasiecka, Charlottesville, VA

Giuseppe Mingione, Parma

Alain Miranville, Poitiers

Giovanni Molica Bisci, Reggio Calabria

Hoai-Minh Nguyen, Lausanne

Kanishka Perera, Melbourne, FL

Patrizia Pucci, Perugia

Dušan Repovš, Ljubljana

Jean Van Schaftingen, Louvain-la-Neuve

Zhi-Qiang Wang, Logan, UT

Binlin Zhang, Harbin

Wenming Zou, Bejing 
ADVANCES IN NONLINEAR ANALYSIS publishes very selected research contributions devoted to nonlinear problems coming from different areas, with particular reference to those introducing new techniques capable of solving a wide range of problems. It especially welcomes contributions dealing with calculus of variations and PDEs, variational and topological methods for ODEs and PDEs, non-smooth analysis and optimization, generalized differentiability, set-valued functions, bifurcation theory, convex analysis or variational inequalities.

All information regarding notes for contributors, subscriptions, Open access, back volumes and orders is available online at www.degruyter.com/ana.

ABSTRACTED/INDEXED IN Baidu Scholar - Clarivate Analytics: Current Contents/Physical, Chemical and Earth Sciences; Journal Citation Reports/ Science Edition; Science Citation Index Expanded; Web of Science · CNKI Scholar (China National Knowledge Infrastructure) · CNPIEC · EBSCO (relevant databases) · EBSCO Discovery Service · Elsevier: SCOPUS · Genamics JournalSeek · Google Scholar · J-Gate · JournalTOCs · KESLINDSL (Korean National Discovery for Science Leaders) · Mathematical Reviews (MathSciNet) · Microsoft Academic $\cdot$ Naviga (Softweco) $\cdot$ Primo Central (ExLibris) · Publons · ReadCube · SCImago (SJR) $\cdot$ Sherpa/RoMEO $\cdot$ Summon (Serials Solutions/ProQuest) · TDNet · Ulrich's Periodicals Directory/ulrichsweb $\cdot$ WanFang Data $\cdot$ WorldCat $($ OCLC) $\cdot$ Zentralblatt Math (zbMATH).

ISSN 2191-9496 · e-ISSN 2191-950X

RESPONSIBLE EDITORS Vicentiu Radulescu, Mathematics Institute "Simion Stoilow" of the Romanian Academy, Bucharest / Department of Mathematics, University of Craiova, 200585 Craiova, Romania.

Email: vicentiu.radulescu@math.cnrs.fr

Marco Squassina, Dipartimento di Matematica e Fisica, Università Cattolica del Sacro Cuore, Via dei Musei 41, I-25121 Brescia, Italy. Email: marco.squassina@dmf.unicatt.it

All email communications related to this journal should be directed to ana.editorial@ degruyter.com

JOURNAL MANAGER Theresa Haney, De Gruyter, Genthiner Straße 13, 10785 Berlin, Germany.

Tel.: +49 (0)30 260 05-375, Fax: +49 (0)30 260 05-250

Email: theresa.haney@degruyter.com

RESPONSIBLE FOR ADVERTISEMENTS Claudia Neumann, De Gruyter, Genthiner Straße 13, 10785 Berlin, Germany.

Tel.: +49 (0)30 260 05-226, Fax: +49 (0)30 260 05-264

Email: anzeigen@degruyter.com

(C) 2018 Walter de Gruyter GmbH, Berlin/Boston

TYPESETTING Dimler \& Albroscheit, Müncheberg

PRINTING Franz X. Stückle Druck und Verlag e.K., Ettenheim

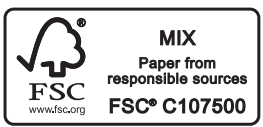




\section{Contents}

César E. Torres Ledesma

Multiplicity result for non-homogeneous fractional

Schrodinger-Kirchhoff-type equations in $\mathbb{R}^{n}-247$

Ahmed Mohammed, Giovanni Porru

Harnack inequality for non-divergence structure

semi-linear elliptic equations -259

Mónica Clapp, Jorge Faya

Concentration with a single sign-changing layer at the higher critical exponents -271

Nils Waterstraat

A remark on bifurcation of Fredholm maps -285

Sheng-Sen Lu

Multiple solutions for a Kirchhoff-type equation with general nonlinearity — 293

Najla Altwaijry, Souhail Chebbi, Hakim Hammami, Pascal Gourdel

Borsuk's antipodal fixed points theorems for compact or condensing set-valued maps -307

Lorenzo D’Ambrosio, Enzo Mitidieri

Uniqueness and comparison principles for semilinear equations and inequalities in Carnot groups - 313
Nikolaos S. Papageorgiou, Vicenţiu D. Rădulescu, Dušan D. Repovš

$(p, 2)$-equations asymmetric at both zero and infinity -327

Rossella Bartolo, Pablo L. De Nápoli,

Addolorata Salvatore

Infinitely many solutions for non-local problems with

broken symmetry - 353

Vincenzo Ambrosio

Zero mass case for a fractional Berestycki-Lions-type problem - 365

Marina Ghisi, Massimo Gobbino, Alain Haraux

A concrete realization of the slow-fast alternative for a semilinear heat equation with homogeneous Neumann boundary conditions - 375

Giovanni Pisante, Tonia Ricciardi

Sign-changing two-peak solutions for an elliptic free boundary problem related to confined plasmas - 385

Jie Xiao

A trace problem for associate Morrey potentials -407 
